\section{An Action Potential from the Motor Nerves of the Jellyfish Aurellia aurita Lamarck.}

So long ago as 1878 , E. A. Schäfer demonstrated in Aurellia a network of bipolar and tripolar nerve cells the processes of which run in all directions, frequently coming close together at points which have been interpreted as synapses ${ }^{1,2}$. Using Holmes's method of silver staining, I have been able to confirm this histological picture; moreover, the fibres are similar to those of the through-conducting system in the mesenteries of Metridium ${ }^{3}$. These fibres can be seen clearly in living Aurellia under phase-contrast or oblique illumination.

It has long been known that a bridge of tissue between two pieces of Aurellia will conduct a stimulus in either direction, resulting in a contraction in each piece. The ability to see the fibres has made it possible to show that this transmission can occur in either direction even when only a single fibre crosses the bridge. Such conduction does not occur when no nerve fibre can be observed under phase-contrast illumination.

Physiological work has shown ${ }^{3}$ that there is a through-conducting system in Colenterates which behaves in the same way as the nerves of higher animals. The existence of an all-or-nothing response and a refractory period is characteristic of nerve fibres. The above experiment with Aurellia shows that the observed nerves are carrying the excitation. Formerly, there was no rigorous proof of this. It is to be expected, therefore, that one or more impulses with action potentials are propagated through at least some of the fibres at each contraction wave. Direct recording from a living nerve shows that this is actually so.

The fibres of the motor nerve net can be picked up individually with a single electrode of thin platinum wire mounted on a micro-manipulator. With a condenser-coupled amplifier of long time-constant, such a preparation gives one action potential at each spontaneous beat of the bell (see traces reproduced herewith). A majority of fibres tested do not show this action potential ; but one cannot distinguish between fibres that have been damaged by the manipulation and those that may be inactive.

A single electrical stimulus applied to the subumbrella surface gives rise to a contraction of the muscle, and the spread of the contraction to every part of the bell is consistent with the histological arrangement of a network of nerves. In these and other experiments I can find no evidence of polariza-

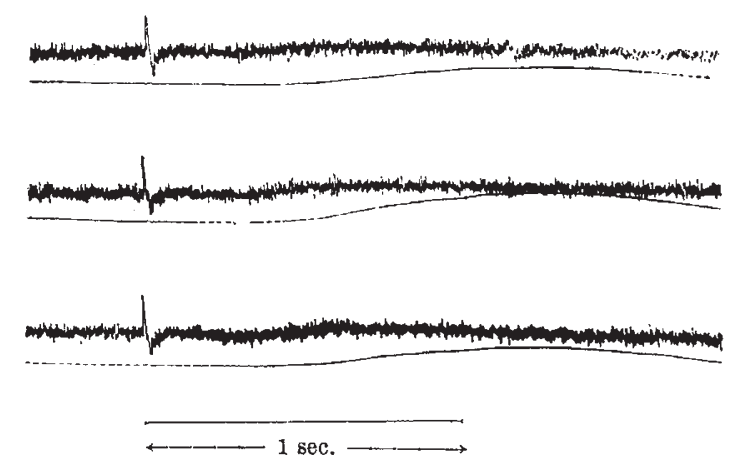

The interval between the nerve impulse in the upper trace and the beginning of the movement shown in the lower is not the
true latent period, since they are not recorded from the same spot tion, interneural facilitation, or decremental conduction in this motor system of Aurellia except occasionally in narrow bridges where it may be due to damage. Direct recording of the impulse in a single nerve fibre shows that the excitation, which can travel in all directions, consists at each beat of a single impulse at any point. This fact gives rise to important conclusions which will be discussed in a later paper.

There is evidence, especially in other Scyphomedusæ, that an excitation can be transmitted across the subumbrella without giving rise to movement en route, and presumably this must go through some diffuse system other than the motor fibres. There is not sufficient evidence to extend these conclusions concerning Aurellia to groups other than Semæostome Scyphozoa.

Department of Zoology,

Adrian Horridge

University of Cambridge. Sept. 4.

${ }^{1}$ Woollard, H. H., and Harpman, J. A., J. Anat., 73, 539 (1939). 2Schäfer, E. A., Phil. Trans. Roy. Soc., 169, 563 (1878).

${ }^{3}$ Pantin, C. F. A., Proc. Roy. Soc., B, 140, 147, Croonian Lecture (1952). - Romanes, G. J., Phil. Trans. Roy. Soc., 167, 269, Croonian Lecture $(1877)$

\section{Competitive Suppression of Prototrophs}

IN a recent publication ${ }^{1}$, Jinks has compounded the erroneous impression, first given by Grigg', that competitive suppression is a neglected source of error in studies of prototrophs or reversions of nutritional requirement in micro-organisms. Kølmark and Westergaard $^{3}$ have shown that Grigg's criticism is inapplicable to their case ; but since Jinks's publication is subsequent to this, and expresses the belief that "it is not easy to see a simple way of avoiding [the] effects" of competitive suppression, a brief recollection of the facts published elsewhere on the matter seems called for. Competitive suppression is certainly a real phenomenon and has been studied experimentally in some detail ${ }^{4-6}$. The findings relevant to the present issue are that: (1) the addition of prototrophs to the culture of organisms from which prototrophs it contains are being screened measures the extent of competitive suppression; (2) when competitive suppression is not acting, dilution of the culture results in a corresponding decrease in the number of prototrophs, and added prototrophs are recovered.

In Nelson's? studies of recombination in $E$. coli, the recovery of added prototrophs showed that competitive suppression was inoperative in a majority of crosses. In that cross in which the parents seemed to prevent the formation of colonies by the prototrophs formed, added prototrophs were not recovered. In these cases, however, suppression was exhibited only in concentrations far beyond those usually employed. Newcombe and $\mathrm{Nyholm}^{8}$, for example, used smaller concentrations, in the range where limited testing showed ten-fold dilutions to give ten-fold decreases in numbers of prototrophs plated (Newcombe, personal communication). Similar consistent relationships between dilution and number of mutants were found in the work of Newcombe and Hawirko ${ }^{9}$ on streptomycin resistance in $E$. coli. Moreover, in their publication they presented evidence for the recovery of added mutants in a background of parents more concentrated than that used in their determination of mutationrates.

Similarly, studies on the reversion of biochemical mutants of Neurospora crassa have been performed 